\title{
Core sets da Classificação Internacional de Funcionalidade, Incapacidade e Saúde
}

\author{
Core sets of the International Classification of Functioning, Disability and Health
}

Core sets de la Clasificación Internacional del Funcionamiento, de la Discapacidad y de la Salud

\section{Marcelo Riberto'}

' Universidade de São Paulo, Faculdade de Medicina de Ribeirão Preto, Departamento de Biomecânica, Medicina e Reabilitação do Aparelho Locomotor. Ribeirão Preto-SP, Brasil.

Submissão: 24-02-2011 Aprovação: 06-09-2011

\section{RESUMO}

A Classificação Internacional de Funcionalidade, Incapacidade e Saúde (CIF) propõe um modelo de entendimento da funcionalidade humana que integra aspectos biomédicos, sociais e pessoais, além de homogeneizar a terminologia que descreve as condições incapacitantes relacionadas à saúde. Todavia, em virtude de sua complexidade e da grande quantidade de aspectos contemplados, foi necessário desenvolver instrumentos práticos baseados nela. Os core sets da CIF são conjuntos de categorias da CIF que descrevem a funcionalidade de pessoas com determinadas condições de saúde. Eles vêm sendo desenvolvidos em processos de consenso com representação multiprofissional e internacional. Os core sets podem ser resumidos ou abrangentes, conforme o uso por apenas um ou mais profissionais. Seu uso permite ao profissional de saúde avaliar aspectos não contemplados por qualquer outro instrumento de avaliação funcional, como os fatores ambientais.

Descritores: Classificação Internacional de Funcionalidade, Incapacidade e Saúde; Estatísticas de sequelas e incapacidades; Avaliação da deficiência; Determinação de necessidades de cuidados de saúde.

\begin{abstract}
The International Classification of Functioning, Disability and Health (ICF) offers a model of human functioning that integrates biomedical, social and personal aspects, and standardize the terminology that describes disabling conditions related to health. However, because of its complexity and large number of aspects to be covered, the development of practical ICF-based tools was needed. ICF core sets are collections of ICF categories that describe functioning of people with determined health conditions. They have been developed in consensus processes with multiprofessional and international representation. They can be either brief or comprehensive, according to its use by one or more professionals. Their use allows clinicians to assess aspects that are not cared about by any other evaluation tool, like environmental factors.
\end{abstract}

Key words: International Classification of Functioning, Disability and Health; Statistics on sequelae and disability; Disability evaluation; Health care needs assessment.

\section{RESUMEN}

La Clasificación Internacional del Funcionamiento, de la Discapacidad y de la Salud (CIF) propone un modelo de comprensión Del funcionamiento humano que integra los aspectos biomédicos, sociales y personales, y estandarizar la terminología que describe las condiciones de discapacidad relacionadas con la salud. Sin embargo, debido a su complejidad y gran número de aspectos cubiertos, fue necesario desarrollar herramientas prácticas basadas en ella. Los core sets son conjuntos de categorías de la CIF que describen el funcionamiento de las personas con ciertas afecciones de salud. Se han desarrollado en procesos de consenso con representación multidisciplinar e internacional. Pueden ser cortos o amplios, de acuerdo con el uso de sólo uno o más profesionales. Su uso permite al profesional de la salud evaluar aspectos no cubiertos por cualquier otra herramienta, como los factores ambientales.

Palabras clave: Clasificación Internacional del Funcionamiento, de la Discapacidad y de la Salud; Estadísticas de secuelas y discapacidad; Evaluación de la discapacidad; Evaluación de necesidades. 


\section{INTRODUÇÃO}

O modelo de entendimento da funcionalidade humana

A Classificação Internacional de Funcionalidade, Incapacidade e Saúde (CIF) ${ }^{(1)}$ foi publicada pela Organização Mundial de Saúde (OMS) em 2001 e fundamenta-se num modelo de entendimento da funcionalidade e da incapacidade que integra os modelos biomédico ${ }^{(2)}$ e social ${ }^{(3-5)}$. Segundo esse modelo, a funcionalidade de um indivíduo com uma determinada condição de saúde depende de aspectos de corpóreos, bem como de fatores pessoais e ambientais(1). Assim como os demais membros da Família Internacional de Classificações da Organização Mundial de Saúde ${ }^{(6)}$, a CIF tem por objetivo a homogeneização de terminologias na área de saúde, permitindo a comparabilidade de dados entre locais e momentos históricos diferentes. As informações colhidas a partir do uso da CIF são, portanto, destinadas a coleta sistemática de dados na população, permitindo a elaboração de relatórios e estatísticas de saúde pública a respeito de condições funcionais relacionadas à saúde que podem vir a ser usadas para políticas públicas para esse setor, mas também para securidade social, trabalho, justiça entre outros ${ }^{(7)}$.

Os componentes desse modelo, bem como o entendimento da sua integração podem ser visualizados na Figura 1.

Os estados de saúde são doenças, transtornos, lesões ou traumas e podem referir-se a outras circunstâncias como enveIhecimento, estresse, anomalias congênitas ou predisposição genética. Pode também incluir informação sobre a patogênese ou etiologia. Em geral, são descritos pela Classificação Internacional de Doenças ${ }^{(8)}$.

Funções do corpo são as funções fisiológicas dos sistemas corpóreos, incluindo as funções psicológicas. As estruturas do corpo são as partes anatômicas do corpo, como órgãos, membros e seus componentes. Anormalidades nas funções ou das estruturas são chamadas deficiências, as quais são definidas como perdas ou alterações significantes (por exemplo, deformidades) de estruturas (como as articulações) e/ou funções (por exemplo, redução da amplitude de movimento, fraqueza muscular, dor e fadiga) ${ }^{(1)}$.

Atividade é a execução de uma tarefa ou ação por um indivíduo e representa a perspectiva individual de funcionalidade. Participação refere-se ao envolvimento de um indivíduo em uma situação de vida e representa a perspectiva social da funcionalidade. Dificuldades para realizar atividade são limitações de atividades (por exemplo, limitação para andar ou carregar objetos). Problemas que um indivíduo pode enfrentar numa situação de vida são conceituados como restrições às participações (por exemplo, na vida comunitária e lazer) ${ }^{(1)}$.
Fatores de contexto representam o pano de fundo global da vida de uma pessoa. Os fatores ambientais englobam aspectos físico, social e de atitudes no qual as pessoas vivem e conduzem suas vidas. Esses fatores são externos ao indivíduo e podem ter influência positiva ou negativa, desempenhando papeis de barreiras ou facilitadores. Fatores pessoais são aspectos particulares da vida de uma pessoa e abrangem características que não são parte da condição de saúde, ou seja, gênero, idade, raça, forma física, estilos e hábitos de vidas e antecedentes sociais. Fatores pessoais não podem estar deficientes, limitados ou restritos. Assim como os fatores ambientais, eles podem modular a funcionalidade positiva ou negativamente ${ }^{(1)}$.

\section{A classificação da funcionalidade}

O modelo acima expõe a estrutura do sistema de classificação da CIF. Como já foi dito anteriormente, o objetivo da classificação é agrupar aspectos semelhantes da funcionalidade humana, organizá-los numa estrutura lógica e defini-los de forma que os termos usados nessa classificação sejam equivalentes no âmbito internacional. A CIF presta-se à classificação da funcionalidade das pessoas e não à classificação das pessoas.

Portanto, os componentes do modelo da funcionalidade (Figura 1) correspondem aos componentes da classificação. Dentro de cada componente, existe uma lista exaustiva de categorias (representadas por códigos), que são as unidades da classificação. As categorias da CIF são hierarquicamente organizadas e sinalizadas por códigos alfanuméricos, elas são dispostas segundo uma organização de tronco-ramo-folha dentro de cada componente.

Com suas 1.454 categorias, a CIF aborda, de forma completa, a funcionalidade humana, esgotando os componentes descritos acima, o que a torna uma classificação extremamente abrangente e significa uma grande vantagem da classificação, porque aumenta seu poder descritivo. Contudo, este também é um dos maiores desafios ao seu uso prático, pois todas as categorias deveriam ser avaliadas em todas as pessoas, o que é impraticável.

Estado de Saúde

(distúrbio ou doença)

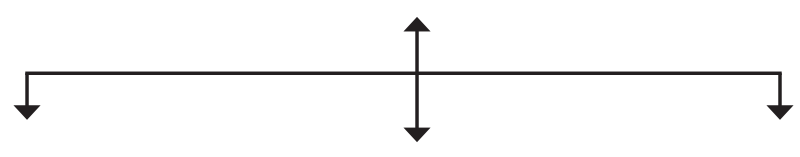

Funções \& Estruturas do Corpo (Deficiências)

Atividades

(Limitações)

Participações

(Restrições)

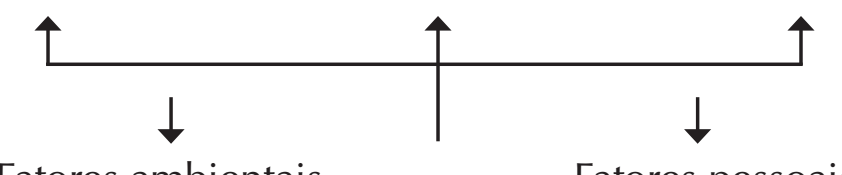

Fatores ambientais

Fatores pessoais

Figura 1 - Modelo integrador da funcionalidade humana segundo a CIF 
Para aumentar a aplicabilidade da classificação, ferramentas embasadas na CIF devem ser desenvolvidas para as necessidades dos usuários ${ }^{(9)}$. Uma solução foi o desenvolvimento dos core sets da CIF.

O objetivo deste texto é enumerar e descrever os core sets da CIF já disponíveis na literatura, discutindo particularidades relativas ao seu uso.

\section{MÉTODO}

Esta é uma revisão narrativa e integradora da literatura. Nas bases de dados Medline e Lilacs foram verificados os artigos nos quais estivessem os termos Classificação Internacional de Funcionalidade, Incapacidade e Saúde, funcionalidade e core set, independentemente da língua de publicação, e publicados desde entre 2001 e 2010. Os artigos poderiam abordar aspectos como o desenvolvimento desses instrumentos, comentários sobre eles, resultados da sua aplicação e testes de validade ou reprodutibilidade. Os resumos foram lidos na versão eletrônica para verificar a adequação ao objetivo do projeto.

\section{RESULTADOS}

Com a estratégia de busca descrita acima, foram identificados 81 artigos, que foram reduzidos para 44 após a leitura dos resumos. Na base Medline, todos os artigos foram posteriores a 2004 e somente três foram escritos em línguas diferentes do inglês (alemão, italiano e norueguês). Na base Lilacs, foram identificados apenas quatro artigos que satisfizeram os critérios de inclusão, todos em português.

\section{DISCUSSÃO}

\section{Core sets da CIF}

O termo core set é da língua inglesa e pode ser traduzido como "conjunto principal" ou "itens essenciais" e refere-se ao conjunto de categorias da CIF que descreve de forma típica a funcionalidade das pessoas com uma determinada condição de saúde. O objetivo do projeto dos core sets da CIF é selecionar as categorias da classificação completa que servem como padrões mínimos para a avaliação e documentação da funcionalidade e saúde em estudos clínicos, encontros clínicos e avaliação multiprofissional abrangente ${ }^{(10)}$. Os primeiros core sets da CIF foram desenvolvidos por uma colaboração entre o escritório para a Família de Classificações Internacionais da OMS (OMS-FIC) no Departamento de Medicina Física e Reabilitação da Universidade Ludwig-Maximilian em Munique (http://www.icf-research-branch.org) e a OMS.

A idéia por trás dos core sets da CIF é que, ao invés de avaliar 1454 aspectos da funcionalidade das pessoas, devem-se avaliar apenas aquelas categorias que são típicas e significativas numa determinada condição de saúde. Desta forma, foram selecionadas apenas 55 a 168 categorias, conforme a condição de saúde, para serem avaliadas em cada paciente (Quadro 1). Os core sets para condições crônicas de saúde podem ser aplicados em qualquer ambiente de atendimento à saúde e refletem todos os aspectos da funcionalidade, considerando a influência do ambiente após a alta hospitalar ${ }^{(10)}$.

Também foram desenvolvidos core sets para situações de saúde que não correspondem a doenças, mas sim a períodos do atendimento a pessoas com alguns grupos de doenças neurológicas, musculoesqueléticas, cardiorrespiratórias nas situações agudas ou subagudas, ou idosos internados (Quadro 1). Para as condições agudas, os core sets apresentam números menores de categorias selecionadas, pois é fácil compreender que a funcionalidade das pessoas nessas condições não inclui uma série de aspectos como vida doméstica, uso de meios de transporte e os fatores ambientais ligados a essas atividades. Todavia, no contexto subagudo, mesmo em se tratando de uma internação de reabilitação, já há o treinamento para essas atividades e as mesmas tornam-se relevantes na descrição da funcionalidade ${ }^{(28-31)}$. Uma ressalva a esses core sets é que eles não são contextualizados para o Brasil, onde a reabilitação na fase subaguda ocorre raramente sob regime de internação, ao contrário de outros países nos quais ocorre a transferência da pessoa com deficiência para instituições ou enfermarias de reabilitação logo após a estabilização do quadro clínico inicial. Há duas propostas de desenvolvimento de core sets da CIF por grupos brasileiros, uma para as afecções musculoesqueléticas relacionadas ao trabalho ${ }^{(36)}$ e ou para indivíduos com síndrome de imunodeficiência adquirida sob terapia antiretroviral ${ }^{(37)}$.

Para cada condição de saúde foram estabelecidos tanto core sets abrangentes como core sets resumidos. Enquanto os core sets abrangentes têm de 55 a 130 categorias, esse número varia de 9 a 39 nos core sets resumidos (Quadro 1). Um core set resumido da CIF para uma condição específica inclui o menor número possível de categorias para ser prático, mas tantos quantos forem necessários para descrever de forma completa o espectro típico de problemas na funcionalidade de pacientes com uma condição específica tanto em estudos como encontros clínicos. Os core sets resumidos têm um compromisso com a praticidade na aplicação e podem ser usados em todos os pacientes incluídos num estudo clínico ou epidemiológico. A princípio, eles poderiam ser aplicados por qualquer profissional de saúde adequadamente treinado. Não foram desenvolvidos core sets resumidos para situações de saúde (Quadro 1).

Por outro lado, os core sets abrangentes da CIF servem para guiar a avaliação multiprofissional em pacientes com uma determinada condição de saúde. Dada a amplitude de aspectos selecionados, que podem inclusive ultrapassar a habilidade do profissional de saúde, é interessante que as categorias do core set abrangente sejam divididas e avaliadas por diferentes membros da equipe multiprofissional. Para fins de exemplificação, os core sets resumidos e abrangentes para lombalgia são apresentados nos Quadros 2 e $3^{\text {(12) }}$.

A OMS recomenda que a cada categoria da CIF seja associado um qualificador que reflita o impacto da condição de saúde sobre aquele aspecto específico da funcionalidade. A escala genérica de qualificadores varia de 0 a 4, conforme a gravidade do comprometimento seguindo uma ordenação nominal, qualitativa, como expresso na coluna central do Quadro $4^{(1)}$. Por outro lado, quando a categoria puder ser avaliada de forma 


\begin{tabular}{|c|c|c|c|c|c|c|}
\hline \multicolumn{2}{|r|}{ Condição de saúde } & $\begin{array}{c}\text { Funções do } \\
\text { corpo }\end{array}$ & $\begin{array}{l}\text { Estruturas } \\
\text { do corpo }\end{array}$ & Atividades e & Fatores & Total \\
\hline \multirow{17}{*}{ 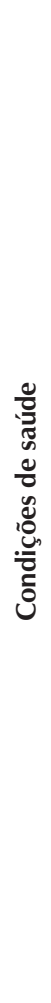 } & Artrite reumatóide ${ }^{(11)}$ & $25(8)$ & $18(7)$ & $32(14)$ & $21(10)$ & $96(39)$ \\
\hline & Lombalgia $^{(12)}$ & $19(10)$ & $5(3)$ & $29(12)$ & $25(10)$ & $78(35)$ \\
\hline & $\mathrm{DCG}^{(13)}$ & $23(10)$ & $1(0)$ & $27(10)$ & $16(6)$ & $67(26)$ \\
\hline & Osteoartrose $^{(14)}$ & $13(3)$ & $6(3)$ & $19(3)$ & $17(4)$ & $55(13)$ \\
\hline & Osteoporose $^{(15)}$ & $15(5)$ & $7(4)$ & $21(6)$ & $26(7)$ & $69(22)$ \\
\hline & Obesidade ${ }^{(16)}$ & $30(3)$ & $18(0)$ & $28(4)$ & $33(2)$ & $109(9)$ \\
\hline & Diabetes mellitus ${ }^{(17)}$ & $36(12)$ & $16(6)$ & $18(5)$ & $29(10)$ & $99(33)$ \\
\hline & $\mathrm{DCIC}^{(18)}$ & $14(10)$ & $1(1)$ & $17(13)$ & $29(12)$ & $61(36)$ \\
\hline & $\mathrm{DPOC}^{(19)}$ & $19(5)$ & $5(3)$ & $24(5)$ & $23(4)$ & $71(17)$ \\
\hline & Depressão ${ }^{(20)}$ & $45(9)$ & $0(0)$ & $48(12)$ & $28(10)$ & $121(31)$ \\
\hline & $A \vee C^{(21)}$ & $41(6)$ & $5(2)$ & $51(7)$ & $33(3)$ & $130(18)$ \\
\hline & Câncer de mama ${ }^{(22)}$ & $22(11)$ & $9(5)$ & $22(11)$ & $23(13)$ & $80(40)$ \\
\hline & Transtornos do sono ${ }^{(23)}$ & $49(5)$ & $8(3)$ & $31(4)$ & $32(3)$ & $120(15)$ \\
\hline & Lesão medular subaguda ${ }^{(24)}$ & $63(8)$ & $14(3)$ & $53(9)$ & $32(5)$ & $162(25)$ \\
\hline & Lesão medular crônica ${ }^{(25)}$ & $44(9)$ & $19(4)$ & $64(11)$ & $41(9)$ & $168(33)$ \\
\hline & Câncer de cabeça e pescoço $\mathrm{O}^{(26)}$ & $34(6)$ & $33(4)$ & $26(6)$ & $19(3)$ & $112(19)$ \\
\hline & Espondilite anquilosante ${ }^{(27)}$ & $23(6)$ & $19(4)$ & $24(7)$ & $14(2)$ & $80(19)$ \\
\hline \multirow{7}{*}{ 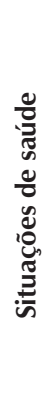 } & Condições cardiorrespiratórias agudas (hospitalar) ${ }^{(30)}$ & 21 & 4 & 10 & 13 & 48 \\
\hline & Condições cardiorrespiratórias subagudas (reabilitação) ${ }^{(32)}$ & 33 & 4 & 23 & 24 & 84 \\
\hline & Condições musculoesqueléticas agudas ${ }^{(31)}$ & 17 & 9 & 11 & 10 & 47 \\
\hline & Condições musculoesqueléticas subagudas ${ }^{(33)}$ & 23 & 7 & 22 & 18 & 70 \\
\hline & Idosos em reabilitação intra-hospitalar aguda ${ }^{(34)}$ & 51 & 14 & 30 & 28 & 123 \\
\hline & Condições neurológicas agudas (intra-hospitalar) & 41 & 5 & 18 & 21 & 85 \\
\hline & Condições neurológicas subagudas (reabilitação) ${ }^{(35)}$ & 54 & 11 & 34 & 17 & 116 \\
\hline
\end{tabular}

Legenda: DCG - dor crônica generalizada, DCIC - doença cardíaca isquêmica crônica, DPOC - doença pulmonar obstrutiva crônica, AVC - acidente vascular cerebral.

\section{Quadro 1 - Quantidade de categorias da CIF selecionadas para cada core set abrangente para condições de saúde (entre parênteses estão expressas as quantidades de categorias selecionadas para os core sets resumidos) e para os core sets de situações de saúde.}

quantitativa, a coluna da direita desse quadro recomenda uma correlação com os qualificadores da CIF. Os qualificadores 8 e 9 representam situações na quais a informação não pode ser obtida (9) ou não o foi de forma adequada (8). Quando se usam os core sets, somente um qualificador deve ser atribuído a cada categoria da CIF.

Um dos aspectos dos core sets da CIF que suscitam muita controvérsia é a forma de pontuar cada uma das categorias. A recomendação dos seus criadores é que somente um qualificador seja associado a cada categoria, exprimindo em que grau a mesma está comprometida. Desta forma, os qualificadores de topografia e natureza da deficiência nas estruturas do corpo não são usados, bem como o qualificador de capacidade nas atividades e participações. Além disso, quando a categoria selecionada é de segundo nível, portanto muito ampla, fica difícil determinar qual dos aspectos a ela relacionados devem ser determinantes. Por exemplo, como ponderar a importância de cada aspecto da categoria d475 (Dirigir), que inclui a condução de automóvel, bicicleta, barco, aeronave ou animal?

É fundamental entender que os core sets da CIF são propostas em construção, no momento atual pode-se afirmar que eles definem o que avaliar, mas não como avaliar. Essa decisão depende do uso da CIF por grupos de trabalho, a fim de se 


\begin{tabular}{|c|c|c|}
\hline \multicolumn{2}{|c|}{ Código da categoria } & Título da categoria \\
\hline \multirow{19}{*}{ 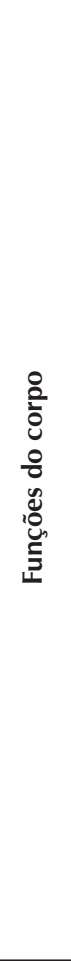 } & b126 & Funções do temperamento e da personalidade \\
\hline & b130 & Funções de energia e de impulsos \\
\hline & b134 & Funções do sono \\
\hline & b152 & Funções emocionais \\
\hline & b180 & Funções de experiência pessoal e do tempo \\
\hline & b260 & Função proprioceptiva \\
\hline & b280 & Sensação de dor \\
\hline & b455 & Funções de tolerância a exercícios \\
\hline & b620 & Funções urinárias \\
\hline & b640 & Funções sexuais \\
\hline & b710 & Funções relacionadas à mobilidade das articulações \\
\hline & b715 & Funções relacionadas à estabilidade das articulações \\
\hline & b720 & Função da mobilidade óssea \\
\hline & b730 & Funções relacionadas à força muscular \\
\hline & b735 & Funções relacionadas ao tônus muscular \\
\hline & b740 & Funções de resistência muscular \\
\hline & b750 & Funções relacionadas ao reflexo motores \\
\hline & b770 & Funções relacionadas ao padrão de marcha \\
\hline & b780 & Sensações relacionadas aos músculos e funções de movimentos \\
\hline \multirow{5}{*}{ 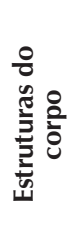 } & s120 & Medula espinhal e estruturas relacionadas \\
\hline & s740 & Estrutura da região pélvica \\
\hline & s750 & Estrutura da extremidade inferior \\
\hline & s760 & Estrutura do tronco \\
\hline & s770 & Estruturas musculoesqueléticas adicionais relacionadas ao movimento \\
\hline
\end{tabular}

Quadro 2 - Categorias dos componentes Funções e Estruturas do corpo selecionadas para o core set abrangente da CIF de lombalgia (as categorias destacadas em negrito pertencem ao core set da CIF de lombalgia resumido).

investigar meios mais adequados de utilização, subsidiando consensos baseados na prática clínica.

A validação dos core sets da CIF faz parte do processo de suas definições, implementação e disseminação. Essa validação vem sendo efetuada empiricamente para diferentes condições e situações de saúde ${ }^{(38,39)}$ e sob a óptica de diferentes categorias profissionais ${ }^{(40-42)}$ ou mesmo de acordo com a visão dos pacientes ${ }^{(43-45)}$.

A avaliação da reprodutibilidade interexaminadores em pacientes com lombalgia mostrou menor concordância de observações para fatores ambientais (32\%) e melhor para funções do corpo (52\%), havendo pouca melhora desses valores com a redução do número de qualificadores para menos categorias resposta ${ }^{(46)}$, o mesmo foi observado com os core sets de artrite reumatóide ${ }^{(47)}$, acidente vascular cerebral( ${ }^{(48)}$, osteoartrite $^{(49)}$ e idosos ${ }^{(50,51)}$. De forma geral, a necessidade percebida é que a operacionalização da aplicação dos core sets da CIF é comprometida pelas definições muito amplas das categorias e que a reprodutibilidade das observações poderá ser muito melhorada com a criação de manuais e diretrizes de utilização, bem como com modificações no sistema de qualificadores $^{(52)}$. Neste sentido, a associação das categorias selecionadas para os core sets com itens de instrumentos já consolidados é um dos caminhos que vêm sendo trilhados com resultados promissores ${ }^{(53)}$. A aplicação do core set da CIF para osteoartrite antes e após a realização de artroplastia total de quadril ou de joelhos mostrou mudanças estatisticamente significantes da freqüência de incapacidade na maior parte das categorias da funcionalidade ${ }^{(49)}$.

Os core sets da CIF não são, todavia, consenso entre o pessoal clínico de reabilitação, pesquisadores e defensores dos direitos da pessoa com deficiência. Ao partirem do princípio de que a funcionalidade pode ser descrita a partir de um conjunto de aspectos característicos de cada doença, eles voltam as costas para o modelo social da funcionalidade que considera fortemente os aspectos ambientais ${ }^{(3-5)}$ e a prática de assistência clínica em reabilitação que enfoca os problemas do paciente, e não no seu diagnóstico ${ }^{(55)}$.

Todavia, a experiência de utilizar um core set da CIF permite ao profissional que o aplica abordar aspectos da funcionalidade que habitualmente não são considerados ou são simplesmente ignorados ${ }^{(56)}$, especialmente no que se refere 


\begin{tabular}{|c|c|c|c|c|c|}
\hline \multicolumn{2}{|c|}{$\begin{array}{l}\text { Código da } \\
\text { categoria }\end{array}$} & \multirow{2}{*}{\begin{tabular}{l}
\multicolumn{1}{c}{ Título da categoria } \\
$\begin{array}{l}\text { Lidar com o estresse e outras demandas } \\
\text { psicológicas }\end{array}$
\end{tabular}} & \multicolumn{2}{|c|}{$\begin{array}{l}\text { Código da } \\
\text { categoria }\end{array}$} & \multirow{2}{*}{$\begin{array}{c}\text { Título da categoria } \\
\text { Produtos e substâncias para consumo pessoal }\end{array}$} \\
\hline \multirow{29}{*}{ 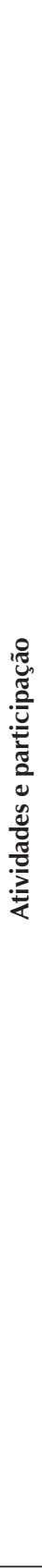 } & d240 & & \multirow{29}{*}{ 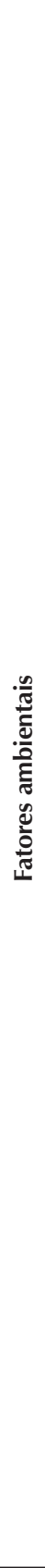 } & e110 & \\
\hline & d410 & Mudar a posição básica do corpo & & e120 & $\begin{array}{l}\text { Produtos e tecnologia para mobilidade e transporte pessoal } \\
\text { em ambientes internos e externos }\end{array}$ \\
\hline & d415 & Manter a posição do corpo & & e135 & Produtos e tecnologia para o trabalho \\
\hline & d420 & Transferir a própria posição & & e150 & $\begin{array}{l}\text { Produtos e tecnologia usados para projeto, arquitetura e } \\
\text { construção de edifícios para uso público }\end{array}$ \\
\hline & d430 & Levantar e carregar objetos & & e155 & $\begin{array}{l}\text { Produtos e tecnologia usados para projeto, arquitetura e } \\
\text { construção de edifícios de uso privado }\end{array}$ \\
\hline & d 445 & Uso da mão e do braço & & $\mathrm{e} 225$ & Clima \\
\hline & d450 & Andar & & e255 & Vibração \\
\hline & d455 & Deslocar-se & & e310 & Família imediata \\
\hline & $\mathrm{d} 460$ & Deslocar-se por diferentes locais & & e325 & $\begin{array}{l}\text { Conhecidos, companheiros, colegas, vizinhos e membros da } \\
\text { comunidade }\end{array}$ \\
\hline & d465 & Deslocar-se usando algum tipo equipamento & & e330 & Pessoas em posição de autoridade \\
\hline & $\mathrm{d} 470$ & Utilização de transporte & & e355 & Profissionais da saúde \\
\hline & $\mathrm{d} 475$ & Dirigir & & e360 & Outros profissionais \\
\hline & d510 & Lavar-se & & e410 & Atitudes individuais de membros da família imediata \\
\hline & d530 & Cuidados relacionados aos processos de excreção & & e425 & $\begin{array}{l}\text { Atitudes individuais de conhecidos, companheiros, colegas, } \\
\text { vizinhos e membros da comunidade }\end{array}$ \\
\hline & d540 & Vestir-se & & e450 & Atitudes individuais de profissionais da saúde \\
\hline & d570 & Cuidar da própria saúde & & e455 & Atitudes individuais dos profissionais relacionados à saúde \\
\hline & d620 & Aquisição de bens e serviços & & $\mathrm{e} 460$ & Atitudes sociais \\
\hline & d630 & Preparação da refeição & & e465 & Normas, práticas e ideologias sociais \\
\hline & d640 & Realização das tarefas domésticas & & e540 & Serviços, sistemas e políticas de transporte \\
\hline & d650 & Cuidar dos objetos da casa & & e550 & Serviços, sistemas e políticas legais \\
\hline & d660 & Ajudar os outros & & e570 & Serviços, sistemas e políticas da previdência social \\
\hline & d710 & Relações interpessoais básicas & & e575 & Serviços, sistemas e políticas de suporte social geral \\
\hline & d760 & Relações familiares & & e580 & Serviços, sistemas e políticas de saúde \\
\hline & $d 770$ & Relações íntimas & & e585 & Serviços, sistemas e políticas de educação e treinamento \\
\hline & d845 & Conseguir, manter e sair de um emprego & & e590 & Serviços, sistemas e políticas de trabalho e emprego \\
\hline & d850 & Trabalho remunerado & & & \\
\hline & d859 & $\begin{array}{l}\text { Trabalho e emprego, outros especificados e não } \\
\text { especificados }\end{array}$ & & & \\
\hline & d910 & Vida comunitária & & & \\
\hline & d920 & Recreação e lazer & & & \\
\hline
\end{tabular}

Quadro 3 - Categorias dos componentes Atividades e participações e Fatores ambientais selecionadas para o core set abrangente da CIF de lombalgia (as categorias destacadas em negrito pertencem ao core set resumido da CIF para lombalgia).

aos fatores ambientais. No entanto, é importante destacar que quando se considera a qualidade de vida relacionada à saúde ou a funcionalidade de acordo com a CIF, as deficiências ainda são o ponto de partida da descrição da experiência vivida pelo indivíduo ${ }^{(57)}$.

Além disso, o uso da CIF sem um guia pode ser fonte de muita variabilidade na descrição da funcionalidade das pessoas e os core sets permitem maior homogeneidade nas observações. No entanto, os core sets da CIF ainda são falhos no que se relaciona à classificação e descrição da funcionalidade de pessoas com mais que uma condição de saúde, pois são estruturados para obtenção de um conjunto específico de características, relacionadas à doença em questão. Na prática clínica, o habitual é que os pacientes tenham mais que um problema de saúde, o que limita o uso dos core sets ou exige flexibilização da sua utilização. 


\begin{tabular}{|c|l|c|}
\hline Qualificador & \multicolumn{1}{|c|}{ Definição nominal } & Definição quantitativa \\
\hline 0 & NENHUM problema (nenhuma, ausente, escasso...) & $0-4 \%$ \\
\hline 1 & Problema LEVE (leve, baixo...) & $5-24 \%$ \\
\hline 2 & Problema MODERADO (médio, regular...) & $25-49 \%$ \\
\hline 3 & Problema GRAVE (elevado, extremo...) & $50-95 \%$ \\
\hline 4 & Problema COMPLETO (total...) & $96-100 \%$ \\
\hline 8 & Não especificada & \\
\hline 9 & Não aplicável & \\
\hline
\end{tabular}

\section{Quadro 4 - Qualificadores genéricos da CIF}

Já se comentou que os core sets da CIF são falhos porque a representação de várias profissões de saúde, especialmente aquelas relacionadas à reabilitação, foi pequena. De fato, o desenvolvimento dos primeiros 12 core sets de condições clínicas específicas contou principalmente com médicos (são as primeiras 12 condições de saúde apresentadas no quadro 2). No entanto, diversos estudos posteriores mostraram a sua validação por diferentes categorias profissionais ${ }^{(40-42)}$.

Por fim, as críticas de que os core sets da CIF não consideram aspectos pessoais nem a satisfação com a saúde na verdade não se direcionam especificamente aos core sets, mas sim à CIF. No seu modelo de entendimento da funcionalidade humana, a CIF destaca a importância dos fatores pessoais (Figura 1), mas na classificação não se aborda esse componente da funcionalidade, pois a divulgação de uma classificação estruturada da funcionalidade das pessoas poderia ser mal-utilizada, resultando em fomento para o preconceito(58).

\section{CONCLUSÃO}

Os core sets da CIF são soluções propostas para a implementação e disseminação do uso da CIF. Eles podem ser usados para finalidades clínicas e de pesquisa, e se referem a condições de saúde ou situações de atendimento. As versões resumidas dos cores sets podem ser aplicadas por apenas um profissional de saúde, enquanto as versões abrangentes devem sê-lo por equipes multiprofissionais, nas quais cada profissional avalia os aspectos mais específicos da sua prática e formação.

Os core sets da CIF são instrumentos em desenvolvimento. Até o momento, há consenso de que eles avaliam 'o que' deve ser observado nos indivíduos com condições específicas de saúde, mas não definem 'como' esses aspectos devem ser qualificados. Há propostas de associação a outros instrumentos de avaliação funcional ou criação de manuais e diretrizes para o seu uso e quantificação.

A principal crítica a respeito dos core sets da CIF é que eles poderiam representar um retorno ao modelo biomédico de entendimento da funcionalidade humana, mas a esta observação contrapõe-se o argumento de que nenhum outro instrumento de avaliação funcional em uso clínico é tão abrangente quanto os core sets da CIF.

\section{AGRADECIMENTO}

Este artigo foi produzido com apoio da Coordenadoria dos Direitos da Pessoa Portadora de Deficiência e do CNPq.

\section{REFERÊNCIAS}

1. Organização Mundial de Saúde. CIF: Classificação Internacional de Funcionalidade, Incapacidade e Saúde. São Paulo: EDUSP; 2003, 325p.

2. Portugal. Secretariado Nacional de Reabilitação. Classificação Internacional de Deficiências, Incapacidades e Desvantagens: um manual de classificação das consequências das doenças (CIDID). Lisboa: SNR/OMS; 1989.

3. Shakespeare T, Watson N. The social model of disability: an outdated ideology. Research in Social Science and Disability. 2002;2:9-28.

4. Hughes B, Paterson K. The social model of disability and the disappearing body: towards a sociology of impairment. Disabil Society 1997;12(3):325-40.

5. Rieser R. The social modal of disability. Invisible children.
In: Joint Conference on Children, Images and Disability, 1995. p.55-6.

6. WHO. The WHO Family of International Classifications. Disponível em http://www.who.int/classifications/en .

7. Farias N, Buchala CM. A Classificação Internacional de Funcionalidade, Incapacidade e Saúde. Conceitos, usos e perspectivas. Rer Bras Epidemiol 2005;8(2):187-93.

8. Organização Mundial de Saúde. CID: Classificação Estatística Internacional de Doenças. São Paulo: EDUSP; 1989.

9. Üstün B, Chatterji S, Konstanisek N. Comments from WHO for the Journal of Rehabilitation Medicine Special supplement on ICF core sets. J. Rehabil. Med. 2004(Suppl 44):7-8.

10. Cieza A, Ewert T, Üstün B, Chatterji S, Konstanjisek N, Stucki G. Development of ICF core sets for patients 
with chronic conditions. J. Rehabil. Med. 2004;(Suppl 44):9-11.

11. Stucki G, Cieza A, Geyh S, Battistella LR, Lloyd J, Symmons D, et al. ICF core sets for rheumatoid arthritis. J. Rehabil. Med. 2004; (Suppl 44):87-93

12. Cieza A, Stucki G, Weigl M, Disler P, Jäckel W, van der Linden $\mathrm{S}$, et al. ICF core sets for low back pain. J. Rehabil. Med. 2004;(Suppl 44):69-74.

13. Cieza, Stucki G, Weigl M, Weigl M, Kullmann L, Stoll T, et al. ICF core sets for chronic widespread pain. J. Rehabil. Med. 2004;(Suppl 44):63-68.

14. Dreinhöfer K, Stucki G, Ewert T, Huber E, Ebenbicher G, Gutenbrunner $\mathrm{C}$, et al. ICF core sets for osteoarthritis. J. Rehabil. Med. 2004;(Suppl 44):75-80.

15. Cieza, Schwarzkopf SR, Sigl T, Stucki G, Melvin J, Stoll $\mathrm{T}$, et al. ICF core sets for osteoporosis. J. Rehabil. Med. 2004;(Suppl 44):81-86.

16. Stucki A, Daansen P, FuessI M, Cieza A, Huber E, Atkinson $\mathrm{R}$, et al. ICF core sets for obesity. J. Rehabil. Med. 2004;(Suppl 44):107-113.

17. Ruof J, Cieza A, Wolff B, Angst F, Ergeleitzis D, Omar Z, et al. ICF core sets for diabetes mellitus. J. Rehabil. Med. 2004;(Suppl 44):100-106.

18. Cieza, Stucki A, Geyh S, Berteanu M, Quittan M, Simon $A$, et al. ICF core sets for chronic ischaemic heart disease. J. Rehabil. Med. 2004;(Suppl 44):94-99.

19. Stucki A, Stoll T, Cieza A, Weigl M, Giardini A, Wever D, et al. ICF core sets for obstructive pulmonary disease. J. Rehabil. Med. 2004;(Suppl 44):114-120.

20. Cieza, Chatterji S, Andersen C, Cantista P, Herceg M, Melvin J, et al. ICF core sets for depression. J. Rehabil. Med. 2004;(Suppl 44):128-134.

21. Geyh S, Cieza A, Schouten J, Dickson H, Frommelt P, Omar Z, et al. ICF core sets for low stroke. J. Rehabil. Med. 2004;(Suppl 44):135-141.

22. Brach M, Cieza A, Stucki G, FüssI M, Cole A, Ellerin BE, et al. ICF core sets for breast cancer. J. Rehabil. Med. 2004;(Suppl 44):121-127.

23. Gradinger F, Cieza A, Stucki A, Michel F, Bentley A, Oksemberg $A$, et al. International Classification of Functioning, Disability and Health (ICF) core sets for persons with sleep disorders: results of the consensus process integrating evidence from preparatory studies. Sleep Med 2010;16 (epub ahead of print).

24. Cieza A, Kirchberger I, Biering-Sorensen F, Baumberger M, Chalifue S, Post MW, et al. ICF core sets for individuals with spinal cord injury in the long-term context. Spinal Cord 2010;48(4):305-312.

25. Kirchberger I, Cieza A, Biering-Sorensen F, Baumberger $M$, Chalifue S, Post MW, et al. ICF core sets for individuals with spinal cord injury in the early post-acute context. Spinal Cord 2010;48(4):297-304

26. Tschiesner U, Rogers S, Dietz A, Yueh B, Cieza A. Development of ICF core sets for head and neck cancer. Head Neck 2010;32(2):210-220.

27. Boonen A, Braun J, van der Horst Bruinsma IE, Huang F, Maksymowych W, et al. ASAS/WHO ICF core sets for ankylosing spondylitis (AS): how to classify the impact of AS on functioning and health. Ann. Rheum. Dis. 2010;69(1):102-7.

28. Stucki G, Üstün B, Melvin J. Applying the ICF for the acute hospital and early post-acute rehabilitation facilities. Disabil. Rehabil. 2005;27(7/8):349-352.

29. Ewert T, Grill E, Bartholomeyczik S, Finger M, Mokrusch $\mathrm{T}$, Konstanjsek $\mathrm{N}$, et al. ICF core set for patients with neurological conditions in the acute hospital. Disabil. Rehabil. 2005;27(7/8):367-373

30. Boldt C, Grill E, Wildner M, Wildner M, Portenier L, Wilke $S$, et al. ICF core set for patients with cardiopulmonary conditions in the acute hospital. Disabil. Rehabil. 2005;27(7/8):375-380.

31. Stoll T, Brach M, Huber EO, Scheuringer M, Schwarzkopf $\mathrm{SR}$, Konstanjsek N, et al. ICF core set for patients with muskuloskeletal conditions in the acute hospital. Disabil. Rehabil. 2005;27(7/8):381-387.

32. Wildner M, Quittan M, Portenier L, Wilke S, Boldt C Stucki G, et al. ICF core set for patients with cardiopulmonary conditions in early post-acute rehabilitation facilities. Disabil. Rehabil. 2005;27(7/8):397-404

33. Scheuringer M, Stucki G, Huber EO, Brach M, Schwartzkopf SR, Kostanjsek N, et al. ICF core set for patients with musculoskeletal conditions in early post-acute rehabilitation facilities. Disabil. Rehabil. 2005;27(7/8):405-410.

34. Grill E, Hermes R, Swoboda W, Uzarewicz C, Kostanjsek N, Stucki G. ICF core set for geriatric patients in early post-acute rehabilitation facilities. Disabil Rehabil 2005;27(7/8):411-417.

35. Stier-Jarmer M, Grill E, Ewert T, Bartholomeyczik S, Finger M, Mokrusch $\mathrm{T}$, et al. ICF core set for patients with neurological conditions in early post-acute rehabilitation facilities. Disabil. Rehabil. 2005;27(7/8):389-395.

36. Lima MAG, Neves RF, Tironi MOS, Nascimento AMDN, Magalhães FB. Avaliação da funcionalidade dos trabalhadores com LER/DORT: a construção do core set da CIF para LER/DORT. Acta Fisiátrica 2008;15(4):229-235.

37. Buchalla CM, Cavalheiro TR. A Classificação Internacional de Funcionalidade, Incapacidade e Saúde e a Aids: uma proposta de core set. Acta Fisiátrica 2008;15(1):42-48

38. Riberto M, Saron TRP, Battistella LR. Resultados do core set da CIF de dor crônica generalizada em mulheres com fibromialgia no Brasil. Acta Fisiátrica 2008;15(1):6-12

39. Castro CLN, Braulio VB, Dantas FAL, Couto APCB. Qualidade de vida em diabetes mellitus e a Classificação Internacional de Funcionalidade, Incapacidade e Saúde: estudo de alguns aspectos. Acta Fisiátrica 2008;15(1):13-17.

40. Hermann KH, Kirchberger I, Stucki G, Cieza A. The comprehensive ICF core set for spinal cord injury from the perspective of occupational therapists: a worldwide validation study using the Delphi technique. Spinal Cord 2010 Nov30 [epub ahead of print].

41. Hermann KH, Kirchberger I, Stucki G, Cieza A. The comprehensive ICF core set for spinal cord injury from the perspective of physical therapists: a worldwide validation study using the Delphi technique. Spinal Cord 2010 Nov30 [epub ahead of print]. 
42. Lemberg I, Kirchberger I, Stucki G, Cieza A. The ICF core set for stroke from the perspective of physicians: a worldwide validation study using the Delphi technique. Eur. J. Phys. Rehabil. Med. 2010;46(3):377-88.

43. Xie F, Thumboo J, Fong K-Y, Lo N-N, Yeo S-J, Yang K-Y, et al. Are they relevant? A critical evaluation of the international classification of functioning, disability and health core sets for osteoarthritis from the perspective of patients with knee osteoarthritis in Singapore. Ann. Rheum. Dis. 2006;65:1067-73.

44. Coenen M, Cieza A, Stamm TA, Amann E, Kollerits B, Stucki G. Validation of the International Classification of Functioning, Disability and Health (ICF) core set for rheumatoid arthritis from the patient perspective using focus groups. Arthitis Res Ther 2006;8(4):R84.

45. Mullis R, Barber J, Lewis M, Hay E. ICF core sets for low back pain: do they include what matters to patients? J. Rehabil. Med. 2007;39:353-7.

46. Hilfiker R, Obrist S, Christen G, Lorenz T, Cieza A. The use of the International Classification of Functioning, Disability and Health core set for low back pain in clinical practice: a reliability study. Physiother. Res. Int. 2009;14(3):147-66.

47. Uhlig T, Lillemo S, Moe RH, Stamm T, Cieza A, Boonen A, et al. Reliability of the ICF core set for rheumatoid arthritis. Ann. Rhem. Dis. 2007;66:1078-84.

48. Starrost K, Geyh S, Trautwein A, Grunow J, CeballosBaumann A, Prosiegel $M$, et al. Interrater reliability of the extended ICF core set for stroke applied by physical therapists. Phys. Ther. 2008;88(7):841-51.

49. Pisoni C, Giardini A, Majani G, Maini M. International classification of functioning, disability and health (ICF) core sets for osteoarthritis. A useful tool in the follow-up of patients after joint arthroplasty. Eur. J. Phys. Rehabil. Med. 2008;44:377-85.
50. Okochi J, Utsunomiya S, Takahashi T. Health measurement using the ICF: test-retest reliability study of ICF codes and qualifiers in geriatric care. Health and Qual Life Outcom 2005;3:46.

51. Stier-Jamer M, Grill E, Müller M, Strobl R, Quittan $M$, Stucki G. Validationof the comprehensive ICF core set for patients in geriatric post-acute rehabilitation facilities. J Rehabil Med 2010 [epub ahead of printing].

52. Grill E, Mansmann U, Cieza A, Stucki G. Assessing observer agreement when describing and classifying functioning with the international classification of functioning, disability and health. J Rehabil med 2007;39:71-76.

53. Kronk RA, Ogonowski JA, Rice CN, Feldman HM. Reliability in assigning ICF codes to children with special health care needs using a developmentally structured interview. Disabil. Rehabil. 2005;27(17):977-83.

54. Cieza A, Hilfiker R, Chatterji S, Kostanjsek N, Üstüm BT, Stucki G. The International Classification of Functioning, Disability and Health could be used to measure functioning. J. Clin. Epidemiol. 2009;62:899-911.

55. College of Occupational Therapists. From interface to integration: A strategy for modernizing occupational therapy services in local health and social care communities. London: College of Occupation Therapists; 2002.

56. McIntyre A, Tempest S. Two steps forward, one step back? A commentary on the disease-specific core sets of the International Classification of Functioning, Disability and Health. Disabil. Rehabil. 2007;29(18):1475-9.

57. Bury M. A comment on the ICIDH2. Disabil. Soc. 2000;15(7):1073-7.

58. Geyh S, Peter C, Müller R, Bickenbach JE, Kostanjsek N, Üstün BT, et al. The personal factors of the International Classification of Functioning, Disability and Health in the literature - a systematic review and content analysis. Disabil. Rehabil. 2010 Oct6 [epub ahead of print] 